\title{
Autismo e Marcadores Precoces do Neurodesenvolvimento
}

\section{Autism and Early Neurodevelopmental Milestones}

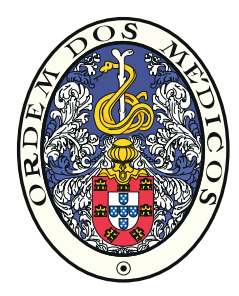

Xavier Ferreira ${ }^{1}$, Guiomar Oliveira $\square^{2,3}$

Acta Med Port 2016 Mar;29(3):168-175 - http://dx.doi.org/10.20344/amp.6790

\section{RESUMO}

Introdução: A perturbação do espectro do autismo, neste texto também designada por autismo, é uma patologia crónica do neurodesenvolvimento, que se manifesta precocemente na infância por alterações da interação social, comunicação e comportamento repetitivo. Não estando disponíveis biomarcadores específicos, o diagnóstico baseia-se exclusivamente na avaliação clínica. O objetivo deste estudo é verificar quais os marcadores precoces do desenvolvimento psicomotor ou neurodesenvolvimento, que se correlacionam significativamente com a gravidade da clínica central do autismo, quocientes de desenvolvimento e com a função adaptativa.

Material e Métodos: Procedemos ao estudo retrospetivo de uma população de 1572 indivíduos com o diagnóstico de autismo seguidos na Unidade de Neurodesenvolvimento e Autismo do Centro de Desenvolvimento da Criança do Hospital Pediátrico do Centro Hospitalar e Universitário de Coimbra. Analisámos seis marcadores precoces do desenvolvimento psicomotor: idades de aquisição 'da marcha', 'das primeiras palavras', 'das primeiras frases', 'do controlo de esfíncter vesical diurno', 'do controlo de esfíncter vesical noturno' e de início das primeiras queixas. Posteriormente dividimos a amostra em três subgrupos de gravidade clínica segundo a escala Childhood Autism Rating Scale e pesquisamos diferenças significativas entre os três subgrupos relativamente aos seis marcadores definidos.

Resultados: O marcador 'idade de aquisição das primeiras frases' foi, de entre os seis, aquele que se correlacionou mais fortemente com as variáveis da clínica de autismo, quocientes de desenvolvimento/inteligência e comportamento ou função adaptativa. Na divisão da amostra em subgrupos de gravidade clínica, foi o de maior gravidade que mostrou idades de aquisição dos marcadores do neurodesenvolvimento mais tardias e idades mais precoces de manifestação das primeiras queixas.

Discussão: Este estudo vem demonstrar a utilidade clinica dos marcadores precoces do desenvolvimento psicomotor nos primeiros anos de vida, como preditores da gravidade da clinica de autismo, cognição e função adaptativa duma vasta população com autismo. Deste modo, estes dados vêm contribuir, em associação com outros, para o prognóstico e previsão da história natural do autismo.

Conclusão: Tendo em conta os resultados deste estudo, recomenda-se fortemente o registo da 'idade de aquisição das primeiras frases' a todos os clínicos que tenham contacto com crianças com autismo.

Palavras-chave: Desenvolvimento da Criança; Perturbação Autística; Perturbações do Desenvolvimento; Prognóstico.

\section{ABSTRACT}

Introduction: Autism spectrum disorder, also referred to in this study as autism, is a neurodevelopmental chronic disease that manifests early in childhood by impairment in social interaction, communication and repetitive behavior. Since there are no specific biomarkers available, the diagnosis is based exclusively on clinical criteria. The purpose of the present study is to determine which are the early psychomotor development or neurodevelopmental milestones that present a significant correlation with the severity of the main symptoms of autism, development quotients, and adaptive function.

Material and Methods: We performed a retrospective study on a sample of 1572 individuals with a diagnosis of autism that were monitored at Hospital Pediátrico do Centro Hospitalar e Universitário de Coimbra, in the Neurodevelopment and Autism Unit. We analyzed six early psychomotor developmental milestones: age of acquisition of 'walking', 'first words', 'first phrases', 'daytime control of bladder sphincter', 'night-time control of bladder sphincter', and age of first complaints. Afterwards, we divided the sample in three subgroups regarding clinical severity, according to the Childhood Autism Rating Scale, and we analyzed significant differences among each other concerning the six milestones established beforehand.

Results: The milestone 'age of first phrases' was, from the six milestones, the one with a stronger correlation with the variables of clinical manifestations of autism, development/intelligence quotients, and adaptive function. In division of the sample into subgroups of clinical severity, it was the most severe that showed later ages of acquisition of the neurodevelopmental milestones and earlier ages of first complaints.

Discussion: This study proves the clinical utility to know the age of achievement of early psychomotor developmental skills, since they act as predictors of clinical severity of autism, cognition, and adaptive function of a wide population with autism. Therefore, this data contribute for prognostic and prediction of autism progression.

Conclusion: Taking into account the results of this study, it is strongly recommended to all clinicians who have contact with autistic children, to record the 'age of acquisition of first phrases'.

Keywords: Autistic Disorder; Child Development; Developmental Disabilities; Prognosis.

\section{INTRODUÇÃO}

A denominação autismo foi utilizada pela primeira vez por Eugen Bleuler, na segunda década do século

XX, para descrever um dos sintomas clínicos principais da esquizofrenia. ${ }^{1}$ Recorreu ao termo grego autós, ${ }^{2}$ que

\footnotetext{
1. Faculdade de Medicina. Universidade de Coimbra. Portugal.

2. Unidade de Neurodesenvolvimento e Autismo. Serviço do Centro de Desenvolvimento da Criança. Centro de Investigação e Formação Clínica. Hospital Pediátrico. Centro Hospitalar e Universitário de Coimbra. Coimbra. Portugal.

3. Clínica Universitária de Pediatria e Instituto de Imagem Biomédica e Ciências da Vida. Faculdade de Medicina. Universidade de Coimbra. Coimbra. Portugal.

$\triangle$ Autor correspondente: Guiomar Oliveira: guiomar@chuc.min-saude.pt

Recebido: 09 de julho de 2015 - Aceite: 26 de outubro de 2015 | Copyright @ Ordem dos Médicos 2016
} 
significa 'o próprio ou por si próprio’3 denunciando o défice na interação social como a sintomatologia central. $\mathrm{O}$ autismo foi mais tarde descrito como entidade clínica/nosológica com início na infância, por Leo Kanner em $1943^{4}$ e Hans Asperger, um ano depois. ${ }^{2,5}$ O autismo é atualmente considerado uma patologia crónica e complexa do neurodesenvolvimento, resultante de disfunção cerebral de etiologia multifatorial, desconhecida em cerca de $80 \%$ dos casos. ${ }^{6}$ Da semiologia típica que o caracteriza faz parte a dificuldade na interação social, na comunicação verbal e não verbal, bem como um padrão de comportamento que se destaca por interesses e atividades restritas e repetitivas. Trata-se de um distúrbio de gravidade clínica variável e por isso denominado na quinta edição do Diagnostic and Statistical Manual of Mental Disorders (DSM-5) (American Psychiatric Association 2013) ${ }^{7}$ como perturbação do espectro do autismo (PEA). Frequentemente, ao quadro semiológico primário associam-se outros sintomas de disfunção neurológica, como o défice intelectual $(68 \%)^{8}$, a epilepsia (até $26 \%)^{9}$, as alterações sensoriais $(95 \%)^{10}$ e o comportamento disruptivo $(23,3 \%),{ }^{7,11,12}$ de entre outras comorbilidades. ${ }^{13}$ Manifesta-se nos primeiros anos de vida, sendo considerado uma das patologias do neurodesenvolvimento mais frequentes, com uma prevalência estimada em Portugal de cerca de uma em cada mil crianças de idade escolar. ${ }^{14,15}$

Trata-se de uma doença com grande implicação na funcionalidade diária do individuo cujo tratamento atual se baseia na intervenção educativa e comportamental, de forma individualizada, e intensiva. 5,16,17 Não se dispondo de biomarcadores, o diagnóstico mantem-se exclusivamente baseado na clínica e o prognóstico, ${ }^{18}$ para além da gravidade intrínseca de cada caso, também está dependente da precocidade da identificação e da qualidade da intervenção. ${ }^{14,19-21}$ Identificar um modelo de neurodesenvolvimento em idades precoces, que nos permita prever o perfil evolutivo ${ }^{18,22}$ em termos de aquisições funcionais, mas também de gravidade, e consequentemente as necessidades de cada criança bem como da respetiva família, é de toda a utilidade clínica para que a intervenção e a expectativa de futuro seja adaptada a cada indivíduo.

Desenhamos este estudo com o objetivo de verificar se os marcadores precoces do neurodesenvolvimento (marcadores de maturação cerebral) e a precocidade das primeiras queixas de autismo se associam com a sua gravidade, com o nível de desenvolvimento global e com a função adaptativa. Analisamos uma larga população com autismo, seguida de um modo longitudinal no Hospital Pediátrico do Centro Hospitalar e Universitário de Coimbra.

\section{MATERIAL E MÉTODOS}

Fazem parte do estudo 1572 indivíduos com o diagnóstico de autismo seguidos na Unidade de Neurodesenvolvimento e Autismo do Serviço do Centro de Desenvolvimento da Criança do Hospital Pediátrico (Hospital do grupo III) do Centro Hospitalar e Universitário de Coimbra. O diagnóstico confirmado por uma equipa multidisciplinar especializada em Neurodesenvolvimento e suas patologias baseou-se num dos instrumentos considerados de gold standard para o efeito: a versão portuguesa da entrevista semi estruturada - Autism Diagnostic Interview ${ }^{\mathrm{TM}}$-Revised (ADI-R) ${ }^{14}$ e no parecer clínico. Analisou-se a dificuldade da relação social através da cotação da área da interação social da ADI-R (escala com cotação de 0 a 30), quanto mais elevado o valor, maior é o défice de interação social.

A classificação da gravidade da clínica global de autismo foi obtida através da aplicação da versão portuguesa da escala Childhood Autism Rating Scale (CARS) ${ }^{14}$ (escala com cotação de 15 a 60), quanto mais elevado for o valor, maior será a gravidade do autismo. Criaram-se três subgrupos de gravidade da clínica global de autismo de acordo com a cotação da escala CARS (ligeiro: < 30, moderado: $\geq$ $30 \leq 36$, grave: $\geq 37$ ).

A avaliação do quociente de desenvolvimento global (QDG) e das subáreas que o compõem: linguagem (QDL), óculo-motora (QDOM), pessoal-social (QDPS) e realização (QDR) foi obtida após a aplicação da escala de desenvolvimento de Ruth Griffiths ${ }^{23}$, por psicólogas experientes em autismo e outras patologias do neurodesenvolvimento. Num subgrupo de 227 sujeitos dispunha-se de dois momentos de avaliação destes parâmetros: na idade pré-escolar e escolar. O valor normal do QDG e subáreas é de $100 \pm 15$; quanto mais elevada a cotação, melhor o nível do desenvolvimento global da criança.

A função adaptativa ou comportamento adaptativo (atividades da vida diária) foi avaliada com o instrumento Vineland Adaptative Behaviour Scale ${ }^{24}$ através da cotação padrão do nível global e das subáreas que o compõem: comunicação, socialização e autonomia. O valor normal da cotação padrão da escala é de $100 \pm 15$; quanto mais elevada a cotação, melhor o nível funcional adaptativo do individuo.

Para além desta avaliação multimodal que por rotina se efetua em todos os doentes é colhida, por pediatra do neurodesenvolvimento, uma história clínica detalhada, sistematizada e armazenada em base de dados digital (Autorização da Comissão Nacional de Proteção de Dados).

Para além das variáveis referentes ao diagnóstico e à gravidade clínica e das diferentes subáreas do QDG e do comportamento adaptativo, foi recolhida informação relativa à idade de aquisição (em meses de idade), referida pelos pais, de marcadores precoces de aquisições chave do neurodesenvolvimento tais como: marcha (primeiros passos sem apoio), primeiras palavras com significado para além de mãe e pai, primeiras frases (compostas de duas ou mais palavras, uma delas verbo, utilizadas funcionalmente no dia a dia), controlo de esfíncter vesical diurno e noturno. Também foi analisada a precocidade das manifestações clínicas de autismo através da variável idade de início (em meses) das primeiras queixas detetadas pelos pais ou profissionais da educação ou saúde.

Foi utilizado o software Statistical Package for the Social Sciences (SPSS, Chicago, IL, EUA), versão para Microsoft Windows ${ }^{\circledR}$ para efetuar a análise estatística.

Recorreu-se ao teste Kolmogorov-Smirnov-Lilliefors 
para testar a distribuição normal das diferentes variáveis e como a maioria evidenciou desvio à normalidade optou-se por utilizar testes de estatística não paramétrica. Para análise da associação linear entre duas variáveis quantitativas recorreu-se ao coeficiente de correlação de Spearman. Considerou-se o nível de significância $(\alpha)=0,05$ $(p<0,05)$. Por fim, para analisar diferenças significativas entre os três subgrupos de gravidade clinica foi utilizado o teste de Kruskal-Wallis, seguido do teste de Mann-Whitney, no caso de o primeiro rejeitar a hipótese nula.

\section{RESULTADOS}

A população é composta por 1572 indivíduos, 1280 $(81,4 \%)$ do género masculino, com uma relação 4,4 rapazes para 1 rapariga. A caracterização clinica está referida na Tabela 1 e a funcional na Tabela 2 .

\section{Correlações}

Para análise da correlação entre duas variáveis quantitativas recorreu-se ao coeficiente de correlação de Spearman. Tendo como base os critérios de Cohen, ${ }^{25}$ os autores

Tabela 1 - Caracterização clínica da população com autismo $(n=1572)$

\begin{tabular}{lccc}
\hline & $\mathbf{n}$ & Mediana & Amplitude interquartil (IQR) \\
\hline Idade das primeiras queixas (meses) & 1341 & 24 & 12 \\
Idade de aquisição da marcha (meses) & 1477 & 14 & 6 \\
Idade de aquisição primeiras palavras (meses) & 1309 & 24 & 22 \\
Idade de aquisição das primeiras frases (meses) & 1007 & 40 & 18 \\
Idade de aquisição do controle esfíncter vesical diurno (meses) & 949 & 36 & 18 \\
Idade de aquisição do controle esfíncter vesical noturno (meses) & 750 & 36 & 12 \\
Idade avaliação CARS (anos) & 1169 & 2,5 & 1 \\
Cotação CARS & 1162 & 34 & 10 \\
Idade avaliação ADI-R (anos) & 1224 & 4,8 & 4 \\
Cotação ADI-R - Interação social & 1367 & 20 & 11 \\
\hline
\end{tabular}

ADI-R: Autism Diagnostic Interview ${ }^{\mathrm{TM}}$-Revised; CARS: Childhood Autism Rating Scale; IQR-Amplitude interquartil.

Tabela 2 - Caracterização do desenvolvimento psicomotor e funcional da população com autismo (n = 1572$)$

\begin{tabular}{|c|c|c|c|}
\hline & $\mathbf{n}$ & Mediana & Amplitude interquartil (IQR) \\
\hline Idade da primeira avaliação do QD (anos) & 1104 & 5,1 & 3,8 \\
\hline Cotação QDG & 1275 & 68 & 42 \\
\hline Cotação QDL & 1099 & 49 & 48 \\
\hline Cotação QDOM & 1101 & 65 & 41 \\
\hline Cotação QDPS & 1101 & 63 & 40 \\
\hline Cotação QDR & 1101 & 73 & 48 \\
\hline Idade de reavaliação do QD (anos) & 227 & 7,3 & 1,8 \\
\hline Cotação QDG reavaliação (reav) & 227 & 79 & 39 \\
\hline Cotação QDL reav & 227 & 76 & 51 \\
\hline Cotação QDOM reav & 226 & 84 & 42 \\
\hline Cotação QDPS reav & 227 & 71 & 32 \\
\hline Cotação QDR reav & 227 & 84 & 38 \\
\hline Idade de avaliação do comportamento adaptativo (anos) & 1348 & 9,3 & 6,5 \\
\hline Comportamento adaptativo - cotação global & 725 & 59 & 26 \\
\hline Comportamento adaptativo - cotação autonomia & 726 & 62 & 25 \\
\hline Comportamento adaptativo - cotação comunicação & 726 & 62 & 33 \\
\hline Comportamento adaptativo - cotação socialização & 726 & 66 & 22 \\
\hline
\end{tabular}

IQR: Amplitude interquartil; QD: Quociente de desenvolvimento; QDG: Quociente de desenvolvimento global; QDL: Quociente de desenvolvimento da linguagem; QDOM: Quociente de desenvolvimento óculo-motor; QDPS: Quociente de desenvolvimento pessoal-social; QDR: Quociente de desenvolvimento de realização; Reav: reavaliação. 
optaram por apresentar apenas os valores de coeficiente de Spearman (coef $\mathrm{S}$ ) de magnitude superior ou igual a 0,30 , independentemente do sinal ser positivo ou negativo. Segundo estes critérios, magnitudes de coef S entre 0,30 e 0,49 representam uma correlação moderada e magnitudes entre 0,5 e 1 representam uma correlação forte. Importa realçar que o sinal de coef $\mathrm{S}$ em nada interfere com a sua magnitude, indicando apenas se o sentido da correlação é direto $(+)$ ou inverso (-).

O marcador precoce de neurodesenvolvimento 'idade de aquisição da marcha', correlacionou-se significativamente $(p<0,05)$ com todas as variáveis de avaliação da clínica de autismo, QD e com o nível de função adaptativa, contudo as magnitudes mais elevadas do coef $\mathrm{S}$ foram obtidas com os QDG $(-0,33)$, QDOM $(-0,33)$ e QDR $(-0,35)$ onde se verificou uma associação moderada negativa.

A 'idade de aquisição das primeiras palavras', correlacionou-se significativamente $(p<0,05)$ com todas as variáveis de avaliação da clínica de autismo, QD e nível de função adaptativa, contudo nenhuma correlação superou a magnitude de 0,3 do coef $S$.

Já a 'idade de aquisição das primeiras frases', correlacionou-se significativamente $(p<0,05)$ com todas as variáveis de avaliação da clínica de autismo, QD e função adaptativa. As magnitudes do coef $S$ foram superiores a 0,30 com associação negativa com o QDG $(-0,35)$, o QDL $(-0,42)$ e com a função adaptativa: comunicação $(-0,41)$, socialização $(-0,31)$ e global $(-0,38)$. Verificou-se uma associação positiva com a gravidade clínica medida pelo défice de interação social da ADI-R $(+0,33)$ e pela escala total da CARS $(+0,30)$.

No subgrupo com reavaliação do QD na idade escolar destacam-se associações negativas moderadas e fortes entre a idade de aquisição das primeiras frases e o QD na idade escolar nomeadamente: QDG reav $(-0,52)$, QDL reav $(-0,61)$, QDOM reav $(-0,42)$, QDPS reav $(-0,47)$ e o QDR reav $(-0,38)$.

Relativamente à 'idade de aquisição do controlo de esfíncter vesical diurno', esta correlacionou-se significativamente $(p<0,05)$ com todas as variáveis de avaliação da gravidade da clínica de autismo, QD e nível de função adaptativa contudo as magnitudes mais elevadas do coef $S$ foram obtidas numa associação negativa com as variáveis QDOM (-0,31) e QDPS (-0,31).

A 'idade de aquisição do controlo de esfíncter vesical noturno', correlacionou-se significativamente $(p<0,05)$ com todas as variáveis de avaliação da clínica de autismo, QD e nível de função adaptativa, exceto com QDOM reav $(p=0,08)$, QDPS reav $(p=0,06)$ e QDR reav $(p=0,15)$.
Porém, não foi obtida magnitude do coef $S$ superior a 0,30.

A 'idade de início das primeiras queixas', correlacionou-se significativamente $(p<0,05)$ com todas as variáveis de avaliação da gravidade clinica do autismo, QD e nível de função adaptativa, exceto com o QDG reav $(p=0,05)$, o QDOM reav $(p=0,12)$, o QDPS reav $(p=0,06)$ e o QDR reav $(p=0,10)$. As magnitudes do coef $S$ superiores a 0,30 obtiveram-se numa associação negativa com a escala CARS $(-0,38)$, e positiva com o QDG $(+0,34)$, o QDL $(+0,31)$, o QDOM $(+0,32)$ e o QDPS $(+0,30)$.

\section{Subgrupos de gravidade clínica de autismo}

O teste de Kruskal-Wallis foi utilizado para analisar os seis marcadores precoces do neurodesenvolvimento nos três subgrupos de gravidade de autismo de acordo com a cotação total da escala CARS (autismo ligeiro, moderado e grave). Analisando os resultados, podemos inferir que em todos os marcadores do neurodesenvolvimento analisados existe pelo menos um subgrupo de gravidade significativamente diferente dos outros dois (Tabela 3 ).

Como o teste de Kruskal-Wallis rejeitou a hipótese nula, foi utilizado o teste de Mann-Whitney entre os três subgrupos de gravidade relativamente à idade de aquisição de cada marcador precoce do neurodesenvolvimento para saber se apenas um subgrupo é significativamente diferente dos outros dois ou se, pelo contrário, os três subgrupos apresentam diferenças significativas entre si.

Relativamente aos marcadores 'idade de aquisição da marcha', 'idade de aquisição das primeiras frases' e 'idade de controlo de esfíncter vesical diurno', os subgrupos mostraram ser significativamente diferentes entre si $(p=0,00$, para todos). Analisando os mean ranks dos subgrupos na tabela 4 podemos concluir que o subgrupo grave tem idades de aquisição dos marcadores de neurodesenvolvimento mais tardias, seguindo-se o moderado, e por fim o ligeiro revelando idades mais precoces de aquisição destas capacidades chave de maturação cerebral.

Relativamente ao marcador 'idade de aquisição das primeiras palavras' os subgrupos mostraram ser significativamente diferentes entre si ( $p=0,00$ nos subgrupos ligeiro e moderado e ligeiro e grave; $p=0,022$ nos subgrupos moderado e grave). Analisando os mean ranks, na tabela 4 podemos concluir que o subgrupo grave apresenta idades de aquisição mais tardias, seguindo-se o moderado e por fim o ligeiro que revela idades mais precoces de atingimento desta capacidade.

Quanto ao item 'idade de controlo de esfíncter vesical noturno', os subgrupos ligeiro e moderado e ligeiro e grave mostraram ser significativamente diferentes $(p=0,00)$.

Tabela 3 - Marcadores precoces de neurodesenvolvimento nos três subgrupos de autismo com diferente gravidade

\begin{tabular}{ccccccc}
\hline & $\begin{array}{c}\text { Idade de aquisição } \\
\text { da marcha }\end{array}$ & $\begin{array}{c}\text { Idade de aquisição } \\
\text { das primeiras } \\
\text { palavras }\end{array}$ & $\begin{array}{c}\text { Idade de aquisição } \\
\text { das primeiras } \\
\text { frases }\end{array}$ & $\begin{array}{c}\text { Idade de controlo } \\
\text { diurno do } \\
\text { esfíncter vesical }\end{array}$ & $\begin{array}{c}\text { Idade de controlo } \\
\text { noturno do } \\
\text { esfíncter vesical }\end{array}$ & $\begin{array}{c}\text { Idade de início das } \\
\text { primeiras queixas }\end{array}$ \\
\hline$\chi^{2}$ & 55,588 & 30,745 & 58,876 & 50,988 & 27,056 & 125,687 \\
$p$ & 0,000 & 0,000 & 0,000 & 0,000 & 0,000 & 0,000 \\
\hline
\end{tabular}


Tabela 4 - Médias dos valores ordenados (mean ranks) das idades respetivas de apresentação de cada marcador relativamente a cada subgrupo

\begin{tabular}{|c|c|c|c|}
\hline & Gravidade autismo & $\mathrm{n}$ & Mean rank \\
\hline \multirow{4}{*}{ Idade de aquisição da marcha } & Ligeiro & 205 & 430,30 \\
\hline & Moderado & 481 & 517,98 \\
\hline & Grave & 391 & 621,85 \\
\hline & Total & 1077 & \\
\hline \multirow{4}{*}{ Idade de aquisição das primeiras palavras } & Ligeiro & 201 & 386,60 \\
\hline & Moderado & 460 & 478,43 \\
\hline & Grave & 283 & 523,86 \\
\hline & Total & 944 & \\
\hline \multirow{4}{*}{ Idade de aquisição das primeiras frases } & Ligeiro & 187 & 291,95 \\
\hline & Moderado & 395 & 376,87 \\
\hline & Grave & 169 & 466,98 \\
\hline & Total & 751 & \\
\hline \multirow{4}{*}{ Idade de controlo diurno do esfíncter vesical } & Ligeiro & 159 & 265,29 \\
\hline & Moderado & 348 & 358,47 \\
\hline & Grave & 199 & 415,28 \\
\hline & Total & 706 & \\
\hline \multirow{4}{*}{ Idade de controlo noturno do esfíncter vesical } & Ligeiro & 134 & 220,95 \\
\hline & Moderado & 277 & 289,64 \\
\hline & Grave & 148 & 315,42 \\
\hline & Total & 559 & \\
\hline \multirow{4}{*}{ Idade de início das primeiras queixas } & Ligeiro & 194 & 641,76 \\
\hline & Moderado & 442 & 568,94 \\
\hline & Grave & 395 & 394,99 \\
\hline & Total & 1031 & \\
\hline
\end{tabular}

Já os subgrupos moderado e grave não evidenciaram diferenças significativas $(p=0,087)$. Dos mean ranks, na Tabela 4 podemos concluir que o subgrupo grave, moderado e ligeiro apresentam idades de controlo de esfíncter vesical noturno progressivamente inferiores.

$\mathrm{Na}$ 'idade das primeiras queixas', os subgrupos ligeiro e moderado, ligeiro e grave, moderado e grave mostraram ser significativamente diferentes ( $p=0,00$, em todos). Da análise dos mean ranks, da Tabela 4 podemos concluir que a manifestação das queixas é mais precoce no subgrupo mais grave, seguido do moderado e por fim do ligeiro.

\section{DISCUSSÃO}

O autismo ou PEA é uma patologia crónica do neurodesenvolvimento cujas manifestações clínicas são muito precoces, tornando-se evidentes, na grande maioria dos casos, antes dos dois anos de idade.

A aquisição das competências motoras que classicamente não se associavam com o desenvolvimento cognitivo, tem-se verificado recentemente que está afetada na população com autismo. ${ }^{22} \mathrm{Na}$ verdade, o início da marcha independente em amostras com autismo é mais tardio do que nos seus pares com desenvolvimento típico, em que o início da marcha surge em média aos 12 meses. ${ }^{26}$

No estudo de Iverson e Wozniak, ${ }^{27}$ a idade média de aquisição da marcha nas crianças com autismo foi de quase 13 meses $(12,59)$ e no grupo controlo de 11,61 meses. $\mathrm{Na}$ nossa população com autismo, a aquisição desta capacidade ocorreu em mediana aos 14 meses. Na verdade, com o nosso trabalho podemos concluir que a aquisição tardia da marcha está associada com menor capacidade intelectual não verbal, representada pelo desenvolvimento das subáreas de realização e óculo-manual onde se verificou um coeficiente de correlação de Spearman negativo de -0,35 e $-0,33$, respetivamente.

A idade em que as nossas crianças adquiriram as primeiras palavras revelou-se um marco com pouco valor clínico. Já a idade em que os pais registaram as primeiras frases correlacionou-se significativamente com todas as variáveis da clínica de autismo, desenvolvimento 
psicomotor e função adaptativa. Na verdade, o aparecimento tardio das primeiras frases associou-se a maior gravidade do autismo quer seja avaliada pela área da interação social da ADI-R $(0,33)$ ou pela cotação da escala CARS $(0,30)$. Falar tarde revelou-se um preditor moderado de menor funcionalidade no comportamento adaptativo a nível global $(-0,37)$, de autonomia $(-0,30)$, comunicação $(-0,41)$ e socialização $(-0,31)$. Em concordância com os nossos resultados, Yang e Jong, ${ }^{28}$ concluem que a ausência de discurso em crianças com autismo aos seis anos, prediz uma restrição grave da função adaptativa na vida adulta. Neste mesmo estudo, os autores referem ainda que as capacidades cognitivas e de comunicação em crianças com autismo no início da idade escolar são as duas variáveis com maior valor prognóstico. A reforçar estes dados Howlin et $a l,{ }^{29}$ num estudo de seguimento de uma população com autismo, referem que os indivíduos com autismo e défices na linguagem revelam pior prognóstico na vida adulta.

A idade em que se registam as primeiras frases também se revelou um importante marcador precoce da capacidade intelectual na idade pré-escolar e escolar. De facto, a associação entre a idade em que as crianças usam frases com duas palavras, e a capacidade intelectual futura, verbal e não verbal, avaliadas através do quociente de desenvolvimento global e subáreas de linguagem, óculo-motor, capacidade pessoal-social e realização, obteve correlações negativas moderadas na idade pré-escolar e fortes na idade escolar. Pode-se concluir que o surgimento tardio das primeiras frases está associado a menor capacidade intelectual verbal e não verbal na idade pré-escolar e sobretudo na escolar, tornando-se assim uma informação muito importante na história clínica (Fig.s 1 e 2). Johnson et al $^{30}$ chegaram a resultados semelhantes, numa amostra de 244 indivíduos, onde associaram problemas na aquisição da linguagem em idades precoces ao défice cognitivo na idade adulta.

Também a idade de aquisição de controlo de esfíncter vesical diurno se revelou um marcador de utilidade clínica.

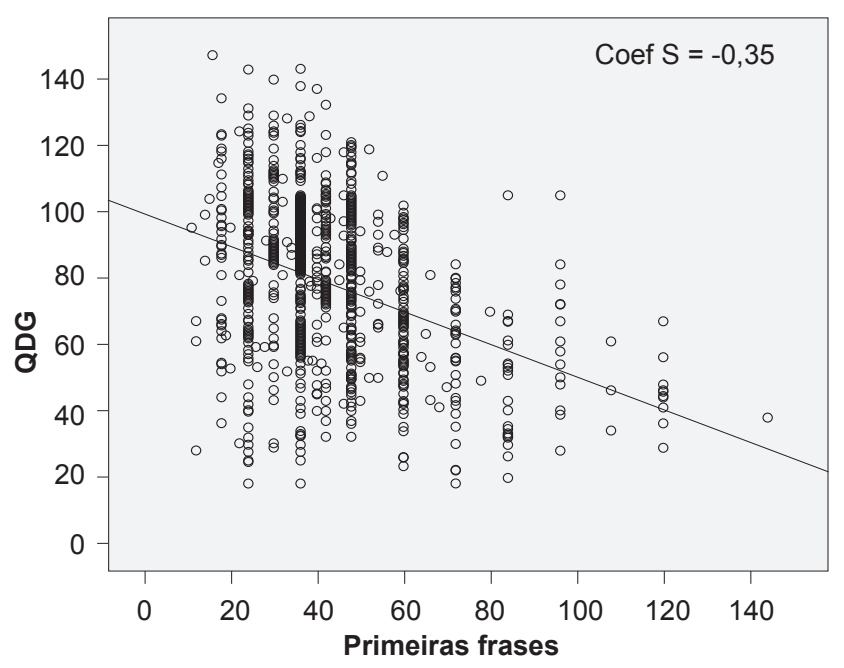

Figura 1 - Relação linear entre a idade de aquisição das primeiras frases e a avaliação do QDG na idade pré-escolar.
Concluiu-se que a aquisição tardia de controlo de esfíncter vesical diurno se associa a menor nível intelectual. Em acordo com estes resultados existem alguns estudos, ${ }^{31,32}$ que associam o sucesso na aquisição de continência de esfíncteres (vesical diurno e noturno e anal) nas crianças com autismo à capacidade intelectual.

Contrariamente ao marcador anterior, a idade de aquisição de controlo de esfíncter vesical noturno, apesar de se ter correlacionado significativamente com quase todas as variáveis estudadas, não se revelou um dado clínico de valor prognóstico. Dalrymple e Ruble ${ }^{32}$ não obtiveram coeficientes de correlação entre as variáveis estudadas, mas dividiram a amostra em subgrupos com base no nível cognitivo e de linguagem, comparando-os, relativamente à idade de aquisição do controlo de esfíncter vesical noturno. Concluíram que os indivíduos com níveis cognitivos mais baixos e menor capacidade verbal tiveram idades de aquisição desta competência significativamente mais tardias.

Tal como noutros quadros patológicos do neurodesenvolvimento, no autismo, as formas mais graves manifestam-se mais precocemente. A idade de início das primeiras queixas evidenciou uma correlação negativa moderada com a gravidade global do autismo, significando que os quadros mais graves se manifestam mais precocemente, permitindo uma fácil identificação pelos prestadores de cuidados.

A idade em que a manifestação do autismo é evidente para os pais tem relação com a capacidade cognitiva da criança. Na verdade, verificamos existir uma correlação positiva entre a idade de início das primeiras queixas e os valores das variáveis QDG $(0,34)$ e subáreas da linguagem $(0,31)$, óculo-motora $(0,32)$ e pessoal-social $(0,30)$, significando que quanto mais precoce a idade de apresentação mais baixa será a capacidade cognitiva. De Giacomo e Fombonne ${ }^{33}$ chegaram à mesma conclusão, num estudo onde é referido que o nível cognitivo se correlaciona fortemente com a idade de apresentação das primeiras queixas. Também Baghdadli et $\mathbf{a l}^{34}$ obtiveram uma correlação

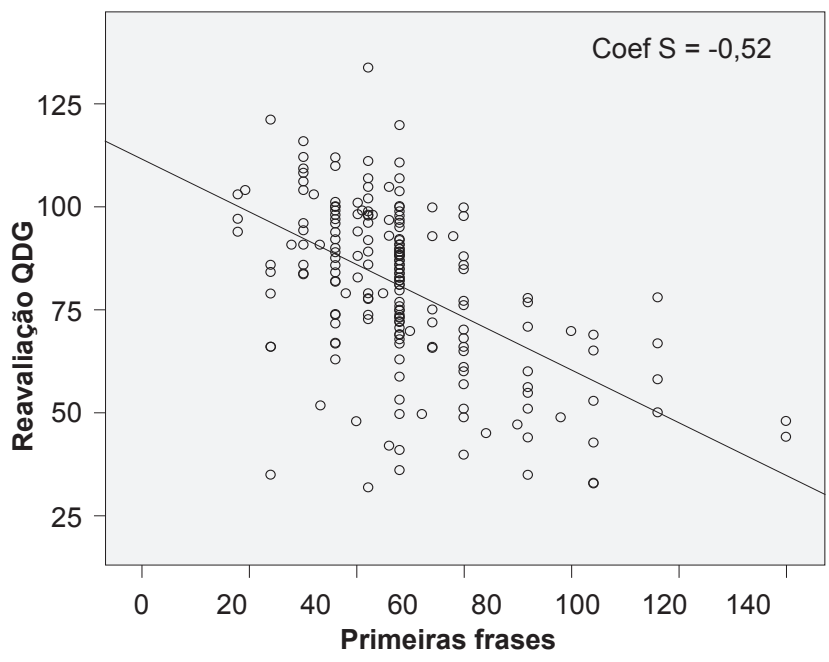

Figura 2 - Relação linear entre a idade de aquisição das primeiras frases e o QDG na idade escolar. 


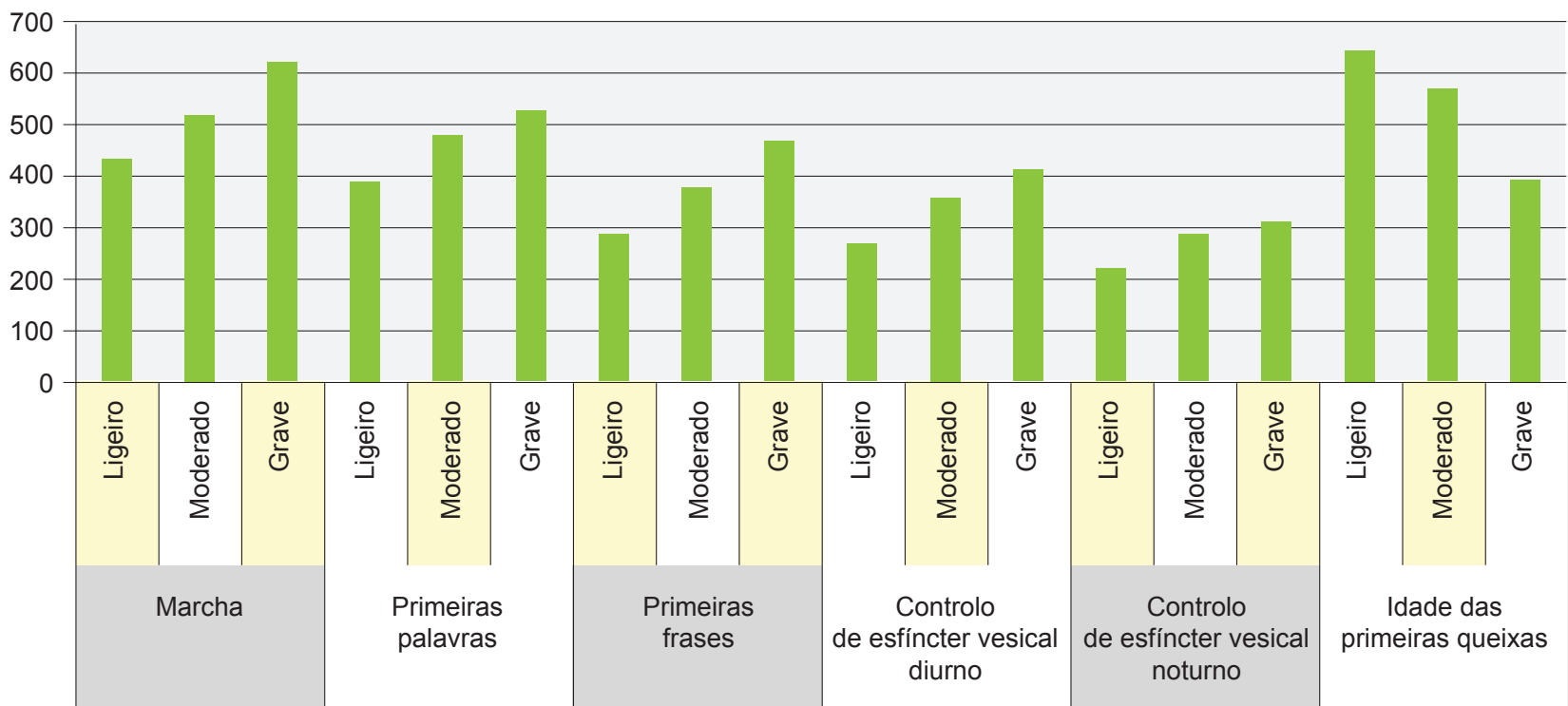

Figura 3 - Médias dos valores ordenados (mean ranks) das idades respetivas de apresentação de cada marcador relativamente a cada subgrupo (divididos com base na gravidade global da clínica de autismo, avaliada pela escala CARS).

estatisticamente significativa entre o nível cognitivo e a idade de manifestação dos primeiros sinais e sintomas.

Tendo em conta os níveis de gravidade clínica com base na escala CARS, os três subgrupos em que dividimos a nossa população diferenciam-se de um modo significativo no que diz respeito à idade de aquisição das capacidades básicas do desenvolvimento psicomotor e na precocidade da manifestação da doença. As crianças que pertenciam ao subgrupo mais grave andaram, falaram e controlaram os esfíncteres mais tarde, acontecendo o oposto no ligeiro, ficando o de gravidade média com idades das aquisições entre os dois extremos (Fig. 3).

Como seria de esperar, a 'idade de início das primeiras queixas', foi mais precoce no subgrupo grave (idade mediana de 15 meses) e mais tardia no subgrupo ligeiro (idade mediana de 24 meses). Estes resultados são consistentes com o estudo de Baghdadli et a ${ }^{34}$ no qual a amostra de indivíduos com autismo foi dividida em dois subgrupos: primeiras queixas antes e depois dos 18 meses, verificando-se que as primeiras queixas antes dos 18 meses se associavam a valores mais baixos do nível cognitivo e do comportamento adaptativo e superiores nas escalas de gravidade da clínica de autismo.

\section{CONCLUSÃO}

Este estudo reforça a utilidade dos registos clínicos cuidadosos da idade de aquisição de etapas chave do desenvolvimento psicomotor como elementos importantes para a história natural do autismo uma vez que se associam com a clínica específica de autismo, mas também com a cognição e a função adaptativa, sobretudo na idade escolar. Podemos então concluir que a idade de aquisição das primeiras frases é um marcador de grande significando clínico em crianças com o diagnóstico de autismo. Assim, apesar de tradicionalmente menos valorizada e menos divulgada do que a idade de aquisição das primeiras palavras, recomendamos fortemente a todos os profissionais de saúde/ clínicos que tenham contacto com estas crianças, o registo adicional da idade de aquisição das primeiras frases. De facto, o seu aparecimento precoce associa-se a melhor potencial intelectual e de função adaptativa na idade escolar e a menor gravidade da clínica específica de autismo.

Uma das limitações deste trabalho, foi o facto de a informação relativa ao aparecimento dos marcadores precoces do neurodesenvolvimento ter sido obtida dos pais, recorrendo à memória, podendo deste modo incorrer em imprecisões. No entanto, o número elevado da população e o facto deste registo ser sistematicamente obtido por clínicos experientes reduzirá tal imprecisão.

Como pontos fortes destacamos o rigor clínico no diagnóstico e avaliação, a dimensão da população e os resultados significativos com valor de associação clínica obtidos entre a competência linguística, a gravidade da sintomatologia nuclear do autismo e a inteligência na idade escolar.

\section{PROTECÇÃO DE PESSOAS E ANIMAIS}

Os autores declaram que os procedimentos seguidos estavam de acordo com os regulamentos estabelecidos pelos responsáveis da Comissão de Investigação Clínica e Ética e de acordo com a Declaração de Helsínquia da Associação Médica Mundial.

\section{CONFIDENCIALIDADE DOS DADOS}

Os autores declaram ter seguido os protocolos do seu centro de trabalho acerca da publicação dos dados de doentes.

\section{CONFLITO DE INTERESSES}

Os autores declaram não ter nenhum conflito de interesses relativamente ao presente artigo.

\section{FONTES DE FINANCIAMENTO}

Os autores declaram não ter recebido qualquer subsídio relativo ao presente artigo. 


\section{REFERÊNCIAS}

1. Parnas J, Bovet P, Zahavi D. Schizophrenic autism: clinical phenomenology and pathogenetic implications. World Psychiatry. 2002;1:131-6.

2. Hans A translated and annotated by FU. 'Autistic psychopathy' in childhood. In: Frith $U$, editor. Autism and Asperger Syndrome. Cambridge: Cambridge University press. 1991;37-92.

3. Dicionário da Língua Portuguesa. Porto: Porto Editora; 2003.

4. Kanner. L. Autistic disturbances of affective contact. Nerv Child. 1943;2:217-50.

5. Oliveira G. Autismo: diagnóstico e orientação Parte | - Vigilância, rastreio e orientação nos cuidados primários de saúde. Acta Pediátrica Port. 2009;40:278-87.

6. Carter M, Scherer S. Autism spectrum disorder in the genetics clinic: a review. Clin Genet. 2013;399-407.

7. American Psychiatric Association. Diagnostic and Statistical Manual of Mental Disorders. 15 ${ }^{\text {th }}$ ed. Arlington, VA: Am Psychiatr Publishing; 2013.

8. Polyak A, Kubina RM, Girirajan S. Comorbidity of intellectual disability confounds ascertainment of autism: implications for genetic diagnosis. Am J Med Genet Part B Neuropsychiatr Genet. 2015;168:600-8.

9. Viscidi EW, Triche EW, Pescosolido MF, McLean RL, Joseph RM, Spence SJ, et al. Clinical characteristics of children with autism spectrum disorder and co-occurring epilepsy. PLoS One. 2013;8:1-11.

10. Tomchek SD, Dunn W. Sensory processing in children with and without autism: a comparative study using the short sensory profile. Am J Occup Ther. 2007;61:190-200.

11. Amr M, Raddad D, El-Mehesh F, Bakr A, Sallam K, Amin T. Comorbid psychiatric disorders in Arab children with Autism spectrum disorders. Res Autism Spectr Disord. 2012;6:240-8.

12. Tarroso MJ, Almeida J, Lontro R, Marques C, Miguel TS, Lobo C, et al. Os efeitos da risperidona nos níveis de prolactina numa amostra de crianças e adolescentes com autismo. Acta Pediátrica Port. 2010;41:111-6.

13. Mannion A, Brahm M, Leader G. Comorbid psychopathology in autism spectrum disorder. Rev J Autism Dev Disord. 2014;1:124-34.

14. Oliveira G. Epidemiologia do autismo em Portugal: um estudo de prevalência da perturbação do espectro do autismo e de caracterização de uma amostra populacional de idade escolar. Coimbra: Universidade de Coimbra; 2005; p.27,28,113,229.

15. Oliveira G. Epidemiology of autism spectrum disorder in Portugal: prevalence, clinical characterization, and medical conditions. Dev Med Child Neurol. 2007;49:726-33.

16. Myers SM, Johnson CP. Management of children with autism spectrum disorders. Pediatrics. 2007;120:1162-82.

17. Crowe B, Salt A. Children and young people on the autism spectrum (NICE Clinical Guideline 170). Arch Dis Child Educ Pract Ed. 2014;1-4.
18. Sutera S, Pandey J, Esser EL, Rosenthal MA, Wilson LB, Barton M, et al. Predictors of optimal outcome in toddlers diagnosed with autism spectrum disorders. J Autism Dev Disord. 2007;37:98-107.

19. Webb S, Jones E. Early identification of autism. Infants Young Child 2009;22(2)100-18.

20. Zwaigenbaum L, Bryson S, Garon N. Early identification of autism spectrum disorders. Behav Brain Res. 2013;251:133-46.

21. Schreibman L. Intensive behavioral/psychoeducational treatments for autism: research needs and future directions. J Autism Dev Disord. 2000;30:373-8.

22. Esposito G, Paşca SP. Motor abnormalities as a putative endophenotype for Autism Spectrum Disorders. Front Integr Neurosci. 2013;7:1-5.

23. Borges P, Pessoa e Costa I, Ferreira C, Gil I, Carvalhão I, Fernandes S, et al. Escalas de Desenvolvimento Mental de Ruth Griffiths - adaptação para a população portuguesa. 2012:922-32.

24. Sparrow S, Balla D, Cicchetti D. Vineland adaptative Behaviour Scales: Interview edition: Survey form. Circle Pines, MN: American Guidance Service; 1984

25. Pallant J. SPSS Survival Manual: A step by step guide to data analysis using SPSS. $4^{\text {th }}$ ed. Adelaide: Midland Typesetters; 2011.

26. Lissauer T, Clayden G. Illustrated textbook of paediatrics. $4^{\text {th }}$ ed. Amsterdam: Elsevier Health Sciences; 2011.

27. Wozniak R, Iverson J. Variation in vocal-motor development in infant siblings of children with autism. J Autism Dev Disord. 2012;37:158-70.

28. Yang $\mathrm{P}$, Jong $\mathrm{Y}-\mathrm{J}$, Hsu H-Y, Chen C-S. Preschool children with autism spectrum disorders in Taiwan: follow-up of cognitive assessment to early school age. Brain Dev. 2003;25:549-54.

29. Howlin P, Savage S, Moss P, Tempier A, Rutter M. Cognitive and language skills in adults with autism: a 40-year follow-up. J Child Psychol psychiatry. 2014;55:49-58.

30. Johnson CJ, Beitchman JH, Brownlie EB. Twenty-year follow-up of children with and without speech-language impairments: family, educational, occupational, and quality of life outcomes. Am J SpeechLanguage Pathol. 2010;19:51-65.

31. Kroeger KA, Sorensen-Burnworth R. Toilet training individuals with autism and other developmental disabilities: a critical review. Res Autism Spectr Disord. 2009;3:607-18.

32. Dalrymple N, Ruble L. Toilet training and behaviors of people with autism: parent views. J Autism Dev Disord. 1992;22:265-75.

33. De Giacomo A, Fombonne E. Parental recognition of developmental abnormalities in autism. Eur Child Adolesc Psychiatry. 1998;7:131-6.

34. Baghdadli A, Picot MC, Pascal C, Pry R, Aussilloux C. Relationship between age of recognition of first disturbances and severity in young children with autism. Eur Child Adolesc Psychiatry. 2003;12:122-7. 